\title{
Retour d'expérience sur la crue du 12 septembre 2015 à Lodève (Hérault, France) : influence du karst sur les débits de pointe de crue
}

\author{
Pascal BRUNET ${ }^{1}$, Christophe BOUVIER ${ }^{2}$
}

${ }^{1}$ CNRS, HydroSciences Montpellier, UMR5569 - e-mail: brunet@msem.univ-montp2.fr

${ }^{2}$ IRD, HydroSciences Montpellier, UMR 5569 - e-mail: bouvier@msem.univ-montp2.fr

\begin{abstract}
RÉSUMÉ. - Le 12 septembre 2015, les environs de Lodève ont reçu des précipitations exceptionnelles. Les cumuls journaliers ont varié entre 300 et $350 \mathrm{~mm}$ sur les stations situées sur le bassin de la Lergue. La pluie a été caractérisée par une cellule intense ayant produit localement des cumuls de $85 \mathrm{~mm}$ en $30 \mathrm{mn}, 140 \mathrm{~mm}$ en $1 \mathrm{~h}$, et $220 \mathrm{~mm}$ en $2 \mathrm{~h}$. La Lergue, principale rivière du secteur, jaugée au niveau de la ville de Lodève, a enregistré sa crue la plus importante depuis l'installation de la station de mesure, il y a plus de 50 ans. Le bassin de la Lergue étant caractérisé par des plateaux karstiques en amont, et par des terrains peu perméables à l'aval, cet événement a donné l'occasion de mettre en évidence les différentes réponses hydrologiques en fonction de la géologie des sous-bassins, et la capacité des karsts à absorber les pluies. Les bassins situés dans la zone pluvieuse (> $250 \mathrm{~mm}$ en $24 \mathrm{~h}$ ) ont en effet eu des réponses très contrastées selon la géologie du bassin, comme le montrent les débits de pointe de crue reconstitués sur une dizaine de sections des cours d'eau. Les débits de pointe de crue sont dans un rapport de 1 à 4 selon le caractère karstique ou non-karstique des bassins. Les débits de pointe de crue estimés par retour d'expérience ont été finalement comparés à ceux d'autres retours d'expérience réalisés en France, puis aux évaluations fournies par des formules régionales (Crupedix, Bressand-Golossof) ou par un modèle pluie-débit (SCS-LR), afin de réviser les paramètres intervenant dans ces formules ou ce modèle.
\end{abstract}

Mots-clés : crue méditerranéenne, extrême, karst, formules régionales, modélisation

\section{Analysis of the September $12^{\text {th }} 2015$ Lodève (France) flash flood: influence of karsts on flood characteristics}

\begin{abstract}
On September $12^{\text {th }}$ 2015, an exceptional rainfall hit the Lodève area (South Of France). The 24-hours cumulated rainfalls ranged from 300 to $350 \mathrm{~mm}$ in most parts of the Lergue watershed. At the hourly time step, an intense rain cell was observed over a restricted area, where rainfall amounts reached $85 \mathrm{~mm}$ in $30 \mathrm{~min}, 140 \mathrm{~mm}$ in $1 \mathrm{~h}$ and $220 \mathrm{~mm}$ in $2 \mathrm{~h}$. The Lergue River recorded at Lodève its highest discharge since the beginning of the observations, more than 50 years ago. The Lergue watershed is characterized by karstic plateau upstream and by poorly permeable Permian sandstone downstream. Thus, it was possible to compare hydrological responses of both units. This flashflood analysis was based from operating gauging station (in Lodève), a post-flood survey and rainfall data from pluviograph and radar images. The watersheds affected by the rainy area $(>250 \mathrm{~mm})$ show very contrasted hydrological response depending on the geological units. Such differences show the ability of karstic watershed to reduce flashflood by lowering and delaying the peak discharge. Finally, these peak flow estimations were compared first to those obtained for other similar events in France, and second to those calculated by regional formulas of extreme discharge prediction or rainfall-runoff model, in order to validate/update such formulas and model.
\end{abstract}

Key-words: Mediterranean flood, extreme event, karst, regional estimation, distributed modelling

\section{INTRODUCTION}

Les crues-éclair du sud-est de la France sont particulièrement redoutables, en raison de leur forte intensité et de leur rapidité [Gaume et al, 2009 ; Creutin et al, 2009]. Depuis quelques années, la pratique de retour d'expérience (REX) s'est considérablement développée en France et en Europe [Gaume et Bouvier 2004 ; Gaume et Borga, 2008 ; Payrastre et al, 2012] pour évaluer les débits de ces crues extrêmes. Les retours d'expérience consistent à reconstituer les débits atteints lors d'événements exceptionnels, à partir des laisses de crues et des témoignages des riverains. Ces retours d'expérience ont notamment aidé à la réévaluation des débits extrêmes en zone méditerranéenne, et des valeurs de plus 20 $\mathrm{m}^{3} / \mathrm{s} / \mathrm{km}^{2}$ sur quelques $\mathrm{km}^{2}$ ont fréquemment été observées [Gaume et al, 2009]. Une question qui reste cependant posée concerne l'influence des karsts sur les débits extrêmes, les travaux sur le sujet restant parcellaires en région méditerranéenne. Coustau et al. [2012] montrent que la contribution des karsts est relativement tardive par rapport à la pointe de crue, et dans ce cas, affecte surtout les volumes ruisselés, mais peu la pointe de crue. Jourde et al. [2007] indiquent toutefois que la rapidité et le volume de cette contribution dépend de l'état de remplissage du karst. Payrastre et al. [2012] attribuent au caractère karstique du bassin le fait que les débits de la Nartuby ont été relativement plus faibles que ceux des bassins environnants lors de l'événement exceptionnel de juin 2010. D'autres systèmes karstiques, comme celui de la ville de Nîmes peuvent avoir une réaction très rapide et venir aggraver fortement le phénomène de crue [Maréchal et al, 2008]. 
L'événement pluvieux intense qui s'est produit les 12-13 septembre 2015 apporte un complément intéressant à ce sujet, parce qu'il a couvert à la fois des plateaux karstiques (partie des Grands Causses) et des terrains sédimentaires peu perméables à l'aval de ces plateaux. Un retour d'expérience a permis de caractériser les débits de pointe de crue d'une dizaine de sous-bassins, de superficie variant de quelque $\mathrm{km}^{2}$ à quelques dizaines de $\mathrm{km}^{2}$, pour moitié en zone karstique et pour moitié en zone non karstique. Ces estimations de débit ont été complétées par quelques observations disponibles issues des stations hydrométriques locales. Cet article présente les données recueillies et les évaluations de débit par retour d'expérience. Ces estimations sont ensuite comparées entre elles, pour contrôler leur homogénéité spatiale, puis interprétées en fonction de la géologie dominante des bassins. Elles sont finalement utilisées pour évaluer les formules régionales pour le calcul des débits extrêmes, ainsi que les paramètres d'un modèle pluie-débit distribué.

\section{EVÈNEMENT MÉTÉOROLOGIQUE}

Les 12 et 13 septembre 2015, des remontées d'air chaud et humide de Méditerranée ont généré des cellules orageuses qui se sont heurtées au massif des Cévennes et ont formé un système stationnaire de pluies intenses pendant plusieurs heures (Fig. 1). Des pluies orographiques ont débuté dans la nuit du 11 au 12 septembre 2015 sur les montagnes languedociennes. Elles se sont poursuivies jusque dans l'après-midi du 12 avec des intensités modérées. On relève ainsi $110 \mathrm{~mm}$ aux Plans et $54 \mathrm{~mm}$ au Caylar entre 6h et 14h TU le 12/09.

A partir de $15 \mathrm{~h} \mathrm{TU}$, des cellules orageuses se sont développées au pied des reliefs et ont déversé des précipitations

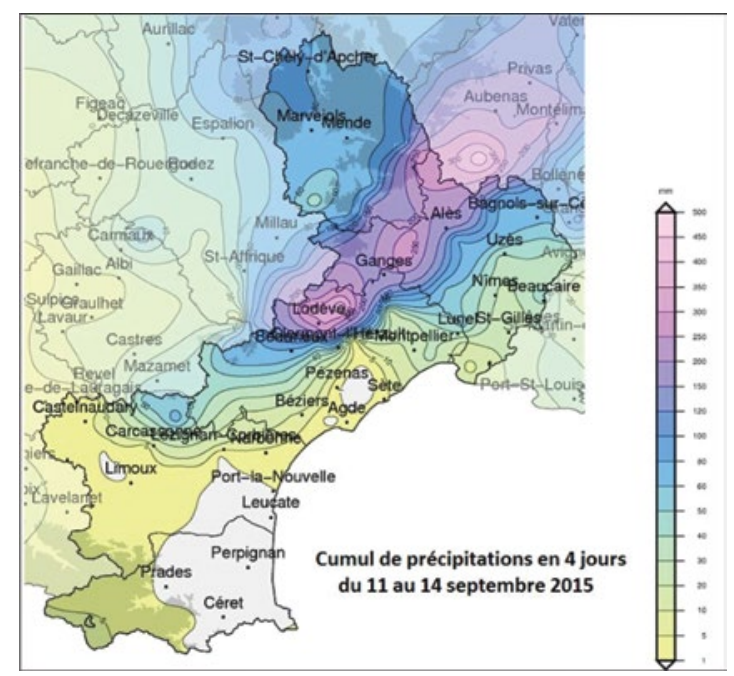

Figure 1 : Pluviométrie régionale du 11 au 14/09 - source MétéoFrance intenses sur le Lodévois avant de se décaler vers le nord-est, la vallée de la Vis et le secteur Ganges Le Vigan. Les intensités relevées dans le secteur ont approché ou dépassé les records français (Tab. 1).

Les cumuls sur 24 heures ont été importants sur une vaste zone autour de Lodève (Fig. 2), tout particulièrement au nord-est, où les cumuls ont été supérieurs à $300 \mathrm{~mm}$. Les cumuls journaliers ont atteint $385 \mathrm{~mm}$ à La Vacquerie, $303 \mathrm{~mm}$ aux Plans, $276 \mathrm{~mm}$ à Soumont, stations situées sur le bassin de la Lergue. A la bordure sud du système précipitant, les pluies ont rapidement diminué, passant de $250 \mathrm{~mm}$ à moins de $50 \mathrm{~mm}$ en moins de $5 \mathrm{~km}$. L'isohyète $250 \mathrm{~mm}$ en $24 \mathrm{~h}$ couvre une superficie de $229 \mathrm{~km}^{2}$, en grande partie sur le bassin de la Lergue. Les cumuls de pluie sur 3 heures montrent la présence d'une cellule intense au sud de Lodève, qui a généré localement des cumuls de $140 \mathrm{~mm}$ en $1 \mathrm{~h}, 220 \mathrm{~mm}$ en $2 \mathrm{~h}$ et plus de $250 \mathrm{~mm}$ en 3 heures (Fig 3). Ainsi, la répartition temporelle des précipitations est-elle contrastée sur l'ensemble de la zone, avec les cumuls en $24 \mathrm{~h}$ les plus importants au nord-est de Lodève, et la présence d'une cellule intense au sud pendant 2-3 heures (Fig. 4).

L'évènement du 12 septembre survient 3 semaines après une crue importante de la Lergue (environ $600 \mathrm{~m}^{3} / \mathrm{s}$ à la confluence) générée par un épisode pluvieux rare à cette période de l'année. Lors de cet épisode, le 23/08, la station de Soumont a enregistré $210 \mathrm{~mm}$ en $24 \mathrm{~h}$, et la crue de la Lergue a concerné essentiellement la partie aval du bassin (au sud de Lodève). Le niveau de remplissage du karst avant le 12/09 était cependant faible. Les données de la source de Ladoux à Pégairolles de l'Escalette, (base ADES http://www.ades.eaufrance.fr/), montrent un étiage peu prononcé de fin d'été (Fig. 5).

\section{CARACTÉRISTIQUES DE LA ZONE}

La Lergue est la principale rivière de la zone touchée par les précipitations. Son bassin versant s'étend sur $182 \mathrm{~km}^{2}$ à Lodève et $428 \mathrm{~km}^{2}$ à la confluence avec l'Hérault, pour une longueur de $18.5 \mathrm{~km}$ à Lodève et $45 \mathrm{~km}$ à la confluence (Fig. 6). Lors de l'événement du 12 septembre 2015, plusieurs rivières languedociennes ont connu des crues importantes comme la Vis et le haut bassin de l'Hérault, mais la Lergue s'est distinguée par les niveaux les plus remarquables et les environs de Lodève ont subi de lourds dégâts.

La géologie du bassin de la Lergue (Fig. 6) est caractérisée à l'amont par les plateaux calcaires jurassiques karstifiés du sud du Larzac. Les vallées (Laurounnet, Lergue, Breze, Soulondre) incisant les plateaux recoupent les terrains plus ou moins argileux du Trias. A l'aval de Lodève, la géologie est constituée de pélites permiennes peu perméables du primaire, roches sédimentaires détritiques argileuses appelées ruffes du Salagou dans la région. L'amont des sous bassins en rive gauche de cette zone (dont Mas d'Alary, Rivernou, Merdanson) présente une géologie complexe à base de calcaires sans caractère karstique marqué.

Tableau 1 : intensités relevées sur les pluviographes de Lodéve (SPC), Soumont et La Vacquerie (Météo France), et valeurs maximum en France métropolitaine d'après http://pluiesextremes.meteo.fr/

\begin{tabular}{|l|c|c|c|c|}
\hline & Lodève & Soumont & La Vacquerie & Max connu Météo France \\
\hline Cumul sur $30 \mathrm{mn}(\mathrm{mm})$ & 75.0 & 64.5 & 85.0 & 100.0 Montélimar 1982 \\
\hline Cumul sur $60 \mathrm{mn}(\mathrm{mm})$ & 140.5 & 119.7 & 112.1 & 111.6 Solenzara 1979 \\
\hline Cumul sur $120 \mathrm{mn}(\mathrm{mm})$ & 221.0 & 185.2 & 167.5 & 184.4 Montpellier 2014 \\
\hline
\end{tabular}




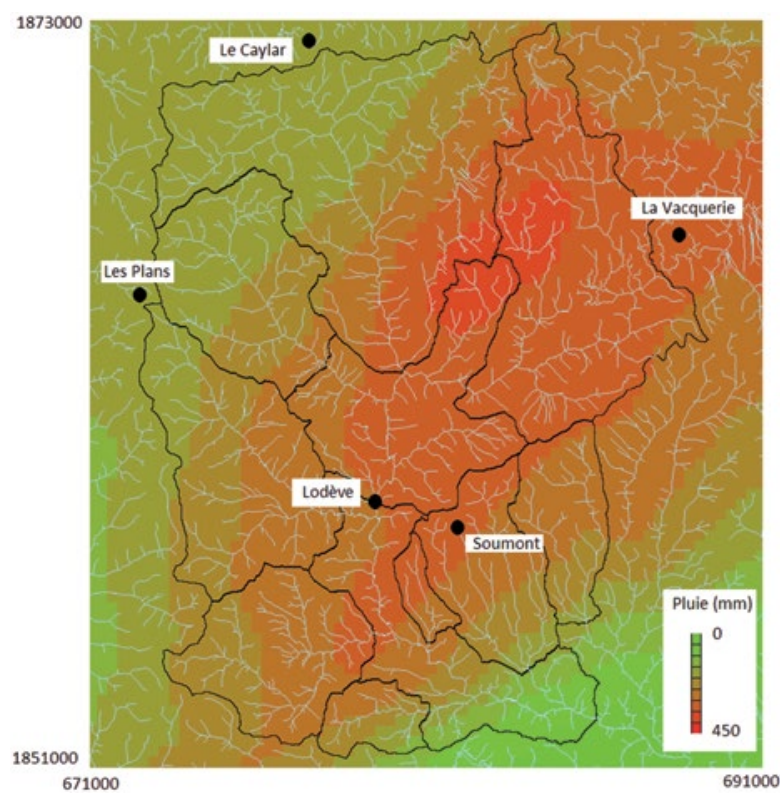

Figure 2 : Répartition spatiale des cumuls de pluie sur $12 \mathrm{~h}$ 12/09/15 $14: 00$ TU au 13/09/15 02:00 TU sur les sous-bassins de la Lergue d'après les données Panthère Meteo-France

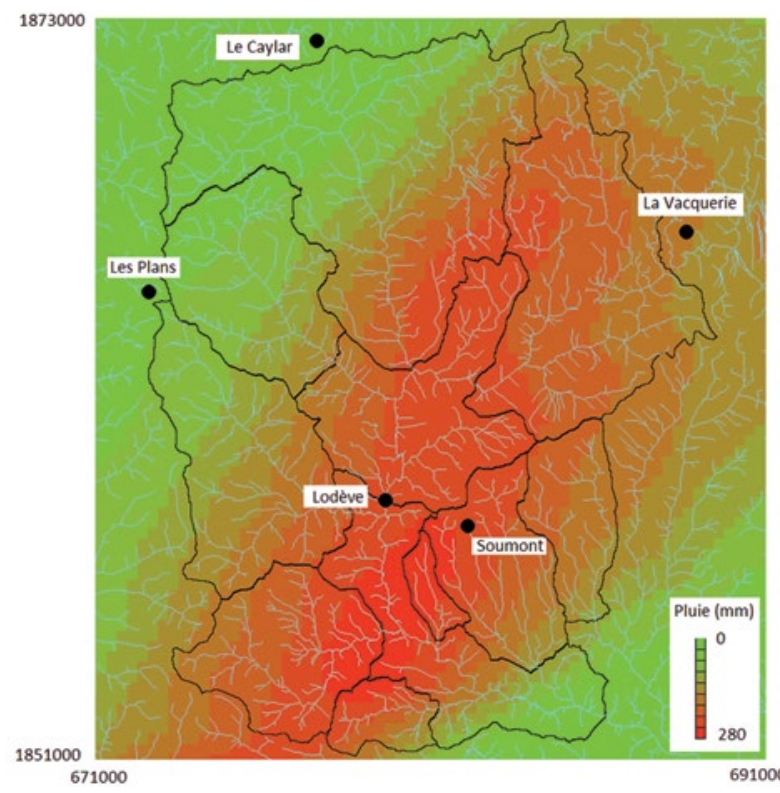

Figure 3 : Répartition spatiale des cumuls de pluie sur 3 , de $15 \mathrm{~h}$ à $18 \mathrm{~h}$ TU le 12/09/2015, d'après les données Panthère Meteo-France

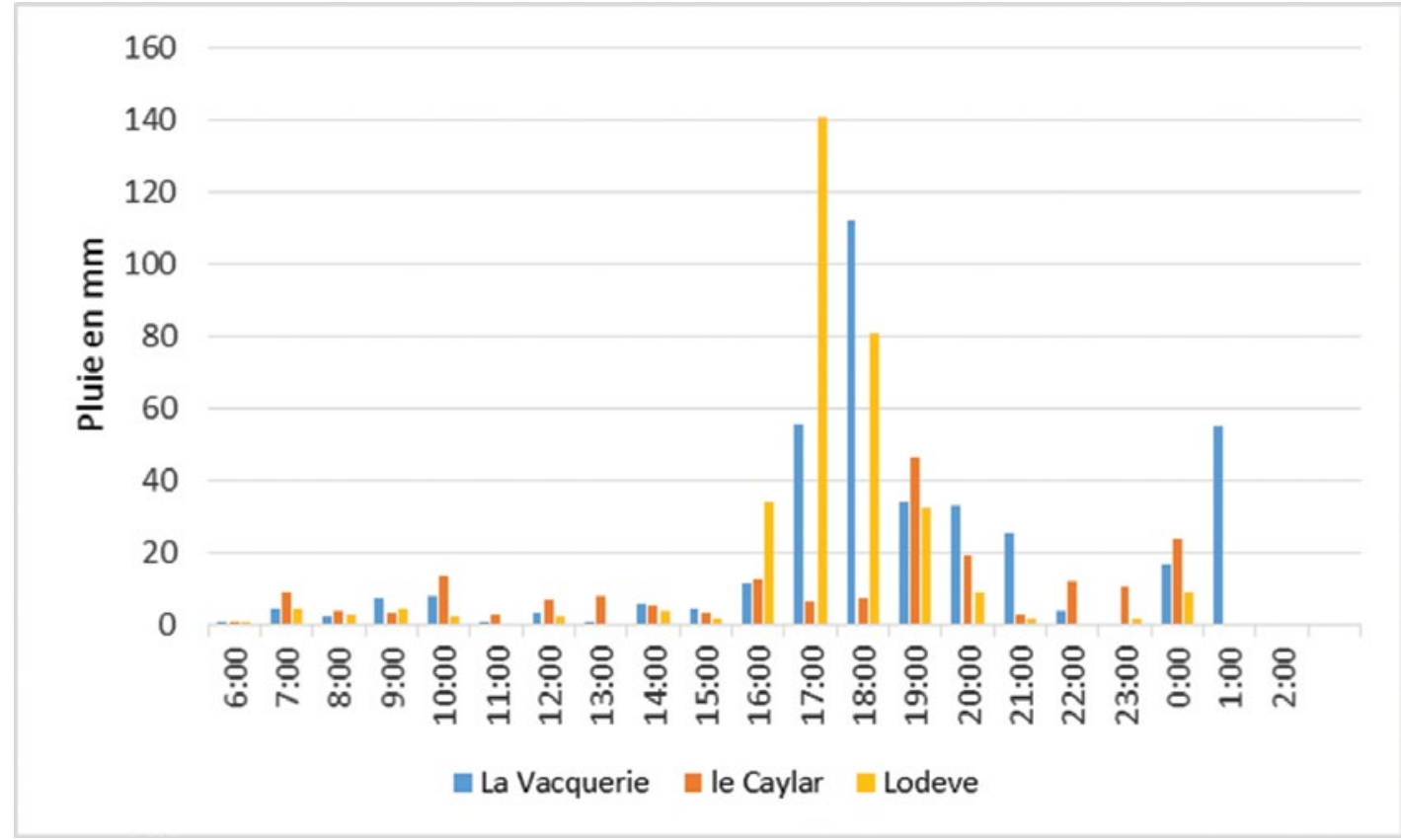

\begin{tabular}{|l|c|c|c|c|c|}
\hline & la Vacquerie & Le Caylar & Les Plans & Soumont & Lodève \\
\hline Cumul max sur 2h & 167 & 66 & 83 & 185 & 221 \\
\hline Cumul sur 24h & 386 & 198 & 303 & 276 & 334 \\
\hline
\end{tabular}

Figure 4 : Répartition horaire des pluies mesurées sur 3 stations pluviométriques du réseau d'observation et cumul sur les 5 stations pluviométriques de la zone. Les cumuls pluviométriques des Plans et du Caylar sont similaires de même entre Soumont et Lodéve.

Tableau 2 : Débits maximums atteints lors des principales crues enregistrées à la station de Lodève selon la courbe de tarage établie à cette station.

\begin{tabular}{|l|c|c|c|c|c|c|c|}
\hline Date & $12 / 09 / 15$ & $31 / 10 / 63$ & $17 / 09 / 14$ & $29 / 04 / 04$ & $18 / 12 / 97$ & $10 / 10 / 87$ & $08 / 11 / 82$ \\
\hline Débit $\left(\mathrm{m}^{3} / \mathrm{s}\right)$ & 595 & 580 & 344 & 312 & 295 & 286 & 210 \\
\hline
\end{tabular}




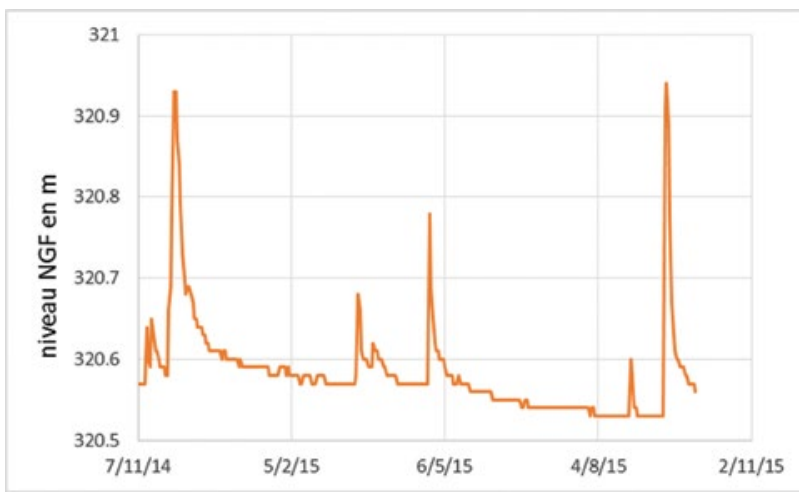

Figure 5: Variation de niveau dans la source de Ladoux en 2014-2015

\section{STATIONS HYDROMÉTRIQUES DU RÉSEAU DE MESURE}

La Lergue est jaugée par le Service de Prévision des Crues (SPC) Med-Ouest au niveau de la ville de Lodève. Depuis le début du fonctionnement de cette station en 1959, la crue de 2015 est la plus forte enregistrée, avec un débit de pointe évalué à $595 \mathrm{~m}^{3} . \mathrm{s}^{-1}$. Seule la crue du 31/10/1963 approche ce débit (Tab. 2). Pour l'épisode de 1963, Météo France indique au Caylar une pluie de $347 \mathrm{~mm}$ en 2 jours dont $227 \mathrm{~mm}$ le 31/10.

L'estimation du débit à Lodève est une extrapolation graphique de la courbe de tarage de cette station. Le plus haut débit jaugé est de $290 \mathrm{~m}^{3} / \mathrm{s}$ en 1964. D'après la hauteur d'eau maximum atteinte et le profil de la section, une vitesse moyenne d'écoulement de $3.4 \mathrm{~m} / \mathrm{s}$ a pu être calculée Cette valeur est importante mais cohérente avec les valeurs obtenues sur les sections aval du retour d'expériences (voir tableau 3). Le débit à Lodève peut paraitre surévalué au regard des débits produits par les affluents amont mais il faut considérer que les

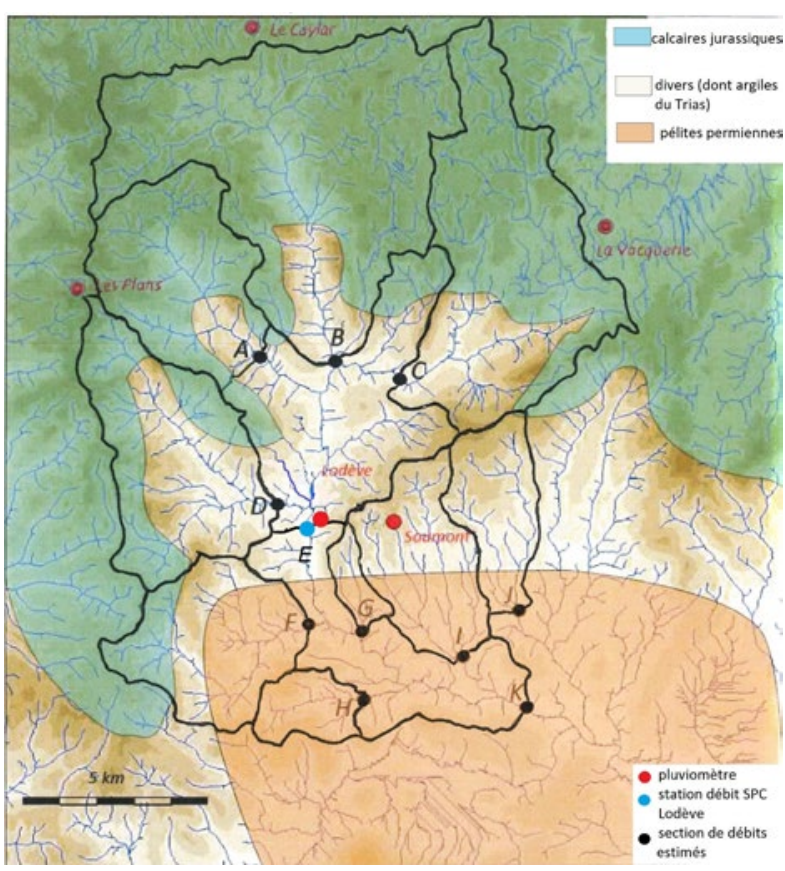

Figure 6 : Localisation des sites de calcul des débits avec géologie simplifiée A - Laurounet, B-Lergue amont, C Brèze, D-Soulondre, E-Lergue à Lodève, $F$-Aubaygues, $G$-Mas d'Alary, H-Roubieu, I-Rivernou, J-Merdanson, $H$ - Roubieu, K--Lergue à Sallèles.

précipitations les plus intenses ont touché les environs immédiats de Lodève.. Les essais de modélisation présentés plus loin sont cohérents avec cette estimation du débit de pointe de crue. La crue est encore amplifiée à l'aval de Lodève par l'apport important des affluents du bassin permien du Salagou (voir tableau 3 et Fig. 6).

Tableau 3 : Caractéristiques et débits des différents sous-bassins de la Lergue. X et Y sont les coordonnées dans la projection Lambert 2 étendue; Geol : géologie dominante sur le bassin (pélites à l'aval signifie que l'amont du bassin est de nature différente : dominante calcaire sans caractère karstique marqué) ; P12h : lame d'eau radar moyenne sur les bassins du 12/09/15 14hTU au 13/09/15 02hTU; P2h : lame d'eau radar moyenne maximale sur $2 h$; $S$ : superficie du bassin; $Q:$ débit estimé lors du retour d'expérience ; Qs : débit spécifique estimé ; Qps : débit pseudo spécifique $=Q / S^{0.8} ; \mathrm{Kr}$ coefficient de Strickler ; I pente retenue pour le calcul du débit ; vitesse moyenne sur la section.

\begin{tabular}{|c|c|c|c|c|c|c|c|c|c|c|c|c|c|}
\hline Bassins & $\begin{array}{l}\text { Index } \\
\text { Fig. } 6\end{array}$ & $X$ & Y & Géol. & $\begin{array}{c}\mathrm{P} 12 \mathrm{~h} \\
\mathrm{~mm}\end{array}$ & $\begin{array}{l}\mathrm{P} 2 \mathrm{~h} \\
\mathrm{~mm}\end{array}$ & $\begin{array}{c}\mathrm{S} \\
\mathrm{km}^{2}\end{array}$ & $\begin{array}{c}\mathrm{Q} \\
\mathrm{m}^{3} / \mathrm{s}\end{array}$ & $\begin{array}{c}\text { Qs } \\
\mathrm{m}^{3} / \mathrm{s} / \mathrm{km}^{2}\end{array}$ & $\begin{array}{c}\text { Qps } \\
\mathrm{m}^{3} / \mathrm{s} / \mathrm{km}^{1.6}\end{array}$ & $\begin{array}{c}\mathrm{Kr} \\
\mathrm{m}^{1 / 3} \cdot \mathrm{s}^{-1}\end{array}$ & $\begin{array}{c}\mathrm{I} \\
\%\end{array}$ & $\begin{array}{c}\mathrm{V} \\
\mathrm{m} \cdot \mathrm{s}^{-1}\end{array}$ \\
\hline Laurounnet & A & 1863003 & 678596 & Karst & 203 & 86 & 22 & 30 & 1.4 & 2.3 & 18 & 1.5 & 3.1 \\
\hline Lergue amont & B & 1862905 & 680790 & Karst & 232 & 89 & 60 & 80 & 1.3 & 3.5 & 18 & 1.0 & 3.2 \\
\hline Breze & $\mathrm{C}$ & 1862356 & 682659 & Karst & 323 & 169 & 42 & 110 & 2.6 & 7.2 & 16 & 0.7 & 2.9 \\
\hline Soulondre & D & 1858656 & 679093 & $\begin{array}{l}\text { Karst/trias } \\
\text { argileux }\end{array}$ & 243 & 131 & 30 & 150 & 5.0 & 9.9 & 18 & 1.0 & 3.4 \\
\hline Lergue Lodève & E & 1858385 & 680085 & $\begin{array}{l}\text { Karst/trias } \\
\text { argileux }\end{array}$ & 266 & 127 & 182 & 595 & 3.3 & 9.7 & & & 3.4 \\
\hline Aubaygues & $\mathrm{F}$ & 1855212 & 679968 & Pélites & 269 & 178 & 20 & 410 & 20.5 & 37.3 & 15 & 1.6 & 4.8 \\
\hline Ru Mas d'Alary & G & 1854946 & 681562 & Pélites à l'aval & 300 & 223 & 2.5 & 45 & 18.0 & 21.7 & 17 & 2.1 & 3.7 \\
\hline Roubieu & $\mathrm{H}$ & 1852956 & 681584 & Pélites & 230 & 197 & 5.5 & 70 & 12.7 & 17.4 & 18 & 1.5 & 2.7 \\
\hline Rivernou & I & 1854114 & 684487 & Pélites à l'aval & 268 & 203 & 15 & 250 & 16.7 & 28.7 & 18 & 2.0 & 4.4 \\
\hline Merdanson & $\mathrm{J}$ & 1855475 & 686165 & Pélites à l'aval & 267 & 159 & 11.5 & 230 & 20.0 & 32.6 & 18 & 0.7 & 3.1 \\
\hline Lergue Sallele & $\mathrm{K}$ & 1852709 & 686468 & Mixte & 261 & 140 & 245 & 1000 & 4.1 & 12.3 & 18 & 0.3 & 2.6 \\
\hline
\end{tabular}




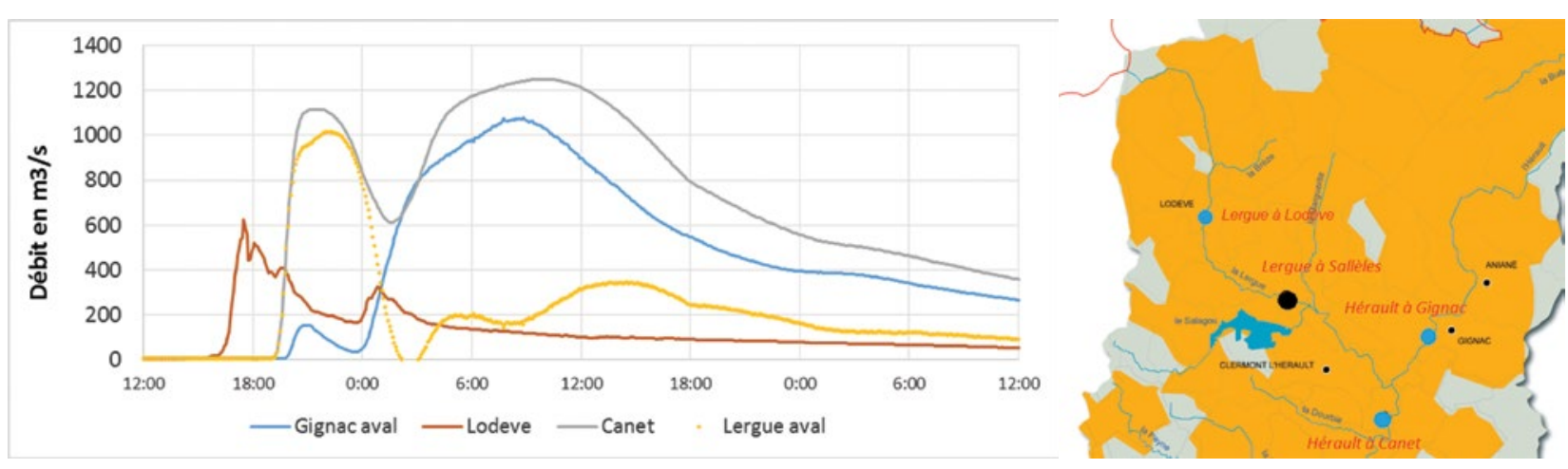

Figure 7 : Localisation et hydrogrammes des stations SPC (les points bleus), de Lodéve sur la Lergue Gignac aval et Canet sur l'Herault et section Rex de Sallèles (point noir). Début le 12/09/16 à 12h TU. Le débit lergue aval est obtenu par soustraction de l'hydrogramme Gignac à celui du Canet.

Aucune autre station n'est en service sur le bassin ou les sous bassins de la Lergue, mais deux stations du Service de Prévision des Crues (SPC) Med-Ouest sont situées sur l'Hérault, en amont et en aval de la confluence avec la Lergue, et ont permis d'enregistrer indirectement la crue sur l'ensemble du bassin de la Lergue (Fig. 7). Cette crue apparait nettement à la station de Canet (aval de la confluence) avec une pointe à $1116 \mathrm{~m}^{3} / \mathrm{s}$ alors que le débit à la station Gignac aval, $3 \mathrm{~km}$ à l'amont de la confluence, ne dépasse pas $150 \mathrm{~m}^{3} / \mathrm{s}$. On peut en conclure que le débit de la Lergue a atteint environ 1000 $\mathrm{m}^{3} / \mathrm{s}$ à la confluence avec l'Hérault, soit un débit spécifique de $2 \mathrm{~m}^{3} / \mathrm{s} / \mathrm{km}^{2}$. Les pointes de crues suivantes (Fig. 7) correspondent aux apports de la Vis et de l'amont du bassin de l'Hérault, hydrauliquement plus éloignés de la confluence et touchés plus tardivement par les pluies.

\section{ESTIMATIONS DES DÉBITS PAR RETOUR D'EXPÉRIENCE}

Une analyse plus poussée de l'événement hydrologique passe par l'étude des réactions des différents sous-bassins versants de la Lergue (Fig. 6). Nous avons dans ce but réalisé des estimations post-crue des débits sur 10 sections de rivière. La méthode utilisée est décrite par Gaume et Borga [2008], et s'appuie sur les principes suivants : 1 / relevé des plus hautes eaux (PHE), des sections en travers et des pentes des lignes d'eau à partir des laisses de crues sur le terrain, 2/ application de formules hydrauliques, type Manning-Strickler, pour le calcul du débit de pointe de crue, 3 / recueil de témoignages pour reconstituer la chronologie de la crue, estimer sa fréquence. Les débits estimés sont affectés d'une incertitude qu'on peut réduire en choisissant soigneusement les sites : traces bien visibles et cohérentes, bief régulier, absence d'obstacle à l'aval. La cohérence spatiale des estimations est également un bon moyen de contrôle. Selon les caractéristiques du site, les incertitudes peuvent être de l'ordre de 20 à $50 \%$. L'estimation du coefficient de rugosité de Strickler est sans doute le principal facteur d'incertitude.

Les sites ont été choisis en zone de karsts, en amont de Lodève, et sur terrains sédimentaires peu perméables, en aval. Les superficies des bassins s'étendent de 2.5 à $245 \mathrm{~km}^{2}$. Neuf bassins ont des superficies inférieures à $60 \mathrm{~km}^{2}$; les 2 bassins dépassant cette superficie sont la Lergue à Lodève $\left(182 \mathrm{~km}^{2}\right)$ et à Salelles $\left(245 \mathrm{~km}^{2}\right)$. Ces bassins ont tous reçu des précipitations dépassant $200 \mathrm{~mm}$, voire 300, mm en 12h (Tab. 3).

Les coefficients de Strickler ont été fixés entre 15 et 18 sur les bassins, en fonction du degré d'encombrement des lits des rivières, et conduisent à des vitesses de l'ordre de 3-4 m.s. ${ }^{-1}$ (Tab.4). Les estimations de débit semblent cohérentes d'un point de vue spatial, en fonction du caractère karstique des bassins considérés. Utiliser le débit pseudo spécifique, débit divisé par la surface élevée à la puissance 0.8 , permet de prendre en compte l'abattement spatial de la pluie et la comparaison des débits est plus fiable pour des bassins de taille différente. Le bassin de l'Aubaygues présente une estimation terrain a priori surestimée car nettement supérieure en débit pseudo spécifique aux autres sections du secteur. Hormis la modification assez radicale du lit de la rivière sur cette section, nous n'avons pas d'explication à ce problème qui illustre les limites des estimations de débits maximum a posteriori. L'estimation post-crue du débit de la Lergue à Salelles, $1000 \mathrm{~m}^{3} / \mathrm{s}$ pour un bassin de $245 \mathrm{~km}^{2}$, est cohérente avec le débit du même ordre, estimé à la confluence avec l'Hérault par différence entre débits amont et aval de la confluence. À l'aval de Salelles, le bassin de la Lergue est composé en rive droite du bassin versant du Salagou dont le débit est régulé par un barrage, et en rive gauche d'une zone peu arrosée (cumul inférieur à $50 \mathrm{~mm}$ ). On peut donc considérer que le débit à Salelles est du même ordre de grandeur que le débit maximum observé plus en aval, à la confluence avec l'Hérault.

Les débits estimés avec des approches comparables montrent une nette différence de comportement entre les bassins versants situés à l'amont de Lodève, comprenant une composante karstique non négligeable et ceux situés à l'aval de la ville, pour lesquels les pélites permiennes imperméables dominent. Les débits spécifiques sont de l'ordre de 1 à $5 \mathrm{~m}^{3} / \mathrm{s} / \mathrm{km}^{2}$ pour les bassins amont et de l'ordre de 12 à $20 \mathrm{~m}^{3} / \mathrm{s} / \mathrm{km}^{2}$ pour les bassins aval, ce qui reste compatible avec les intensités pluviométriques observées (atteindre un débit de $20 \mathrm{~m}^{3} / \mathrm{s} / \mathrm{km}^{2}$ suppose de produire une pluie efficace de $72 \mathrm{~mm} / \mathrm{h}$ sur le temps de concentration du bassin). La différence de comportement remarquable entre les bassins à dominante karstique et les bassins aval doit être pondérée par le fait que les précipitations en $2 \mathrm{~h}$ ont été relativement moins intenses sur les bassins du Laurounnet et de la Lergue amont (voir Tab. 3). On dispose néanmoins d'un point de comparaison fiable avec la Bréze, bassin à dominante karstique touché par des précipitations intenses sur $2 \mathrm{~h}$, et pour lequel le débit pseudo spécifique estimé ne dépasse pas $8 \mathrm{~m}^{3} / \mathrm{s} / \mathrm{km}^{1.6}$, alors que ce débit atteint 17 à $37 \mathrm{~m}^{3} / \mathrm{s} / \mathrm{km}^{1.6}$ pour les bassins en aval de Lodève. Dans ce cas précis la présence de karst a pu diviser par au moins 2, voire 4 le débit de pointe observé par rapport aux débits estimés sur les bassins versants aval. 


\section{Comparaison avec les formules de prédétermination de crues}

On dispose de plusieurs formules pour la prédétermination des débits de crues des bassins non jaugés. On a utilisé dans cette étude la formule Crupedix pour l'estimation des débits décennaux avec un facteur correctif pour les débits exceptionnels et celle dite de "Bressand-Golossof » pour les débits exceptionnels. Les valeurs obtenues ont été comparées aux débits estimés sur le terrain.

Bressand-Golossof : $\mathrm{Q}_{\mathrm{m}^{3} / \mathrm{s}}$ rare $=30 * \mathrm{~S}_{\mathrm{km}^{2}}^{0.75}$ pour des bassins compris entre 20 et $400 \mathrm{~km}^{2}$

$$
\begin{aligned}
& \text { Crupedix : } \mathrm{Q}_{\mathrm{m}^{3} / \mathrm{s}}(10 \mathrm{ans})=\mathrm{S}_{\mathrm{km}^{2}}^{0.8} *\left(\mathrm{P}_{\mathrm{mm}} / 80\right)^{2} * \mathrm{~K} \\
& \mathrm{Q}_{\mathrm{m}^{3} / \mathrm{s}} \text { exceptionnel }=\mathrm{Q}(10 \mathrm{ans}) * \mathrm{~K}_{2}
\end{aligned}
$$

avec $\mathrm{S}$ superficie du bassin, $\mathrm{P}$ pluie journalière décennale, $\mathrm{K}=1$ pour PACA et Corse, $\mathrm{K}=1.5$ pour le Languedoc Roussillon, $\mathrm{K}_{2}=5$ pour PACA et Corse, $\mathrm{K}_{2}=4$ pour le Languedoc-Roussillon.

Pour Lodève, la pluie journalière décennale est de $166 \mathrm{~mm}$ |Météo France, 1999]. Pour cette valeur, les formules de Crupedix « exceptionnel » et de Bressand-Golossof « rare » donnent quasiment les mêmes valeurs de débits pour une surface donnée.

Pour l'Aubaygues, le Rivernou, le Merdanson, les débits de septembre 2015 sont proches des débits rares calculés par les formules Bressand-Golossof ou Crupedix exceptionnel (Fig. 8). Sur les autres bassins, les débits atteignent à peine le débit décennal calculé par Crupedix. Or, la période de retour de ces débits est clairement supérieure à 10 ans, notamment pour la Lergue à Lodève, où la crue est la plus forte depuis le début des observations. Les formules issues de synthèses de données régionales

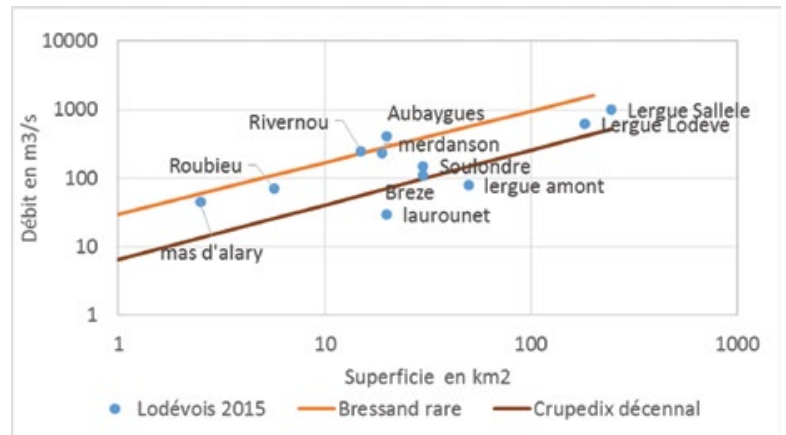

Figure 8 : Position des débits du 12/09/15 par rapport aux formules de prédétermination des crues.

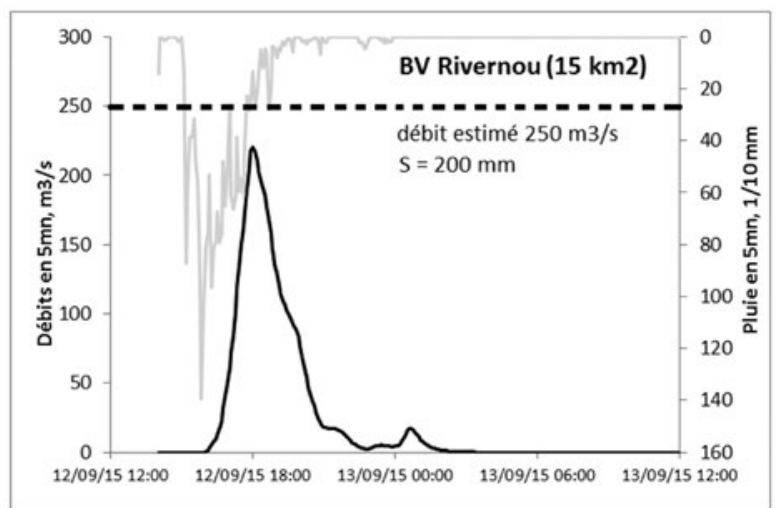

Figure 9 : Simulation de la crue à l'exutoire $d u$ bassin du Rivernou, à l'aide du modèle SCS-LR. Une valeur de $S=200 \mathrm{~mm}$ permet d'approcher le débit estimé à $250 \mathrm{~m}^{3} / \mathrm{s}$ par retour d'expérience. semblent donc manifestement surestimer les débits rares/exceptionnels pour ces bassins amont à composante karstique.

\section{Essais de modélisation des crues}

Les essais de modélisation ont été réalisés à partir du modèle SCS-LR, modèle distribué, événementiel, parcimonieux. Ce modèle opère sur la base de mailles carrées régulières (ici $25 \mathrm{~m}$ ) ; la pluie brute reçue par chaque maille $\mathrm{m}$, $\mathrm{Pb}_{\mathrm{m}}(\mathrm{t})$, est estimée à partir de la pluie radar (au pas de temps $5 \mathrm{mn}$ ) ; la pluie nette $\mathrm{Pn}_{\mathrm{m}}(\mathrm{t})$ (pluie contribuant à l'hydrogramme de crue) est calculée sur chaque maille $\mathrm{m}$ à l'aide de l'expression dérivée du modèle SCS :

$$
P n_{m}(t)=P b_{m}(t) \cdot\left(\frac{P_{m}(t)-0.2 S}{P_{m}(t)+0.8 S}\right) \cdot\left(2-\frac{P_{m}(t)-0.2 S}{P_{m}(t)+0.8 S}\right)
$$

où $\mathrm{S}$ désigne la capacité maximale de stockage en début d'épisode, paramètre du modèle et $\mathrm{P}_{\mathrm{m}}(\mathrm{t})$ le cumul de la pluie reçue par la maille $\mathrm{m}$ depuis le début de l'épisode.

Chaque maille $\mathrm{m}$ produit à l'exutoire du bassin un hydrogramme élémentaire :

$$
q_{m}(t)=\frac{1}{K_{m}} \int_{t_{0}}^{t-T_{m}} P n_{m}(\tau) \cdot \exp \left(-\frac{t-T_{m}-\tau}{K_{m}}\right) d \tau
$$

où $T_{m}$ désigne le temps de propagation de l'écoulement entre la maille et l'exutoire, et $\mathrm{km}$ le temps de diffusion au cours de ce parcours. Les variables $\mathrm{T}_{\mathrm{m}}$ et $\mathrm{km}$ font intervenir 2 paramètres, uniformes sur l'ensemble des mailles : la vitesse d'écoulement Vo $(\mathrm{m} / \mathrm{s})$ et un coefficient Ko, reliant linéairement le temps de propagation $\mathrm{T}_{\mathrm{m}}$ au temps de diffusion $\mathrm{km}: \mathrm{km}=\mathrm{K}_{\mathrm{o}} \cdot \mathrm{T}_{\mathrm{m}}$. Les paramètres ont été fixés à $\mathrm{Vo}_{\mathrm{o}}=2.5 \mathrm{~m} / \mathrm{s}$ et $\mathrm{Ko}=0.7$, valeurs appliquées sur le bassin du Gardon à Anduze (Marchandise, 2007). L'hydrogramme complet de la crue est obtenu en sommant les hydrogrammes élémentaires de toutes les mailles. Ce modèle est implémenté dans la plate-forme ATHYS www.athys-soft.org.

Le calage du modèle se limite donc au seul paramètre $\mathrm{S}$, désignant la capacité maximale de stockage en eau au début de l'épisode. Le calage a été réalisé pour chaque sous-bassin, en minimisant l'écart entre le débit de pointe calculé par le modèle et le débit de pointe estimé par retour d'expérience : les valeurs de $\mathrm{S}$ sont de l'ordre de $100-200 \mathrm{~mm}$ pour les bassins à dominante sédimentaire $(100 \mathrm{~mm}$ pour Aubaygues et Merdanson, $200 \mathrm{~mm}$ pour Mas Alary, Roubieu, Rivernou (Fig. 9) et Lergue à Sallèles), et de $600-700 \mathrm{~mm}$ pour les bassins à dominante karstique (Lergue amont, Breze (Fig. 10),

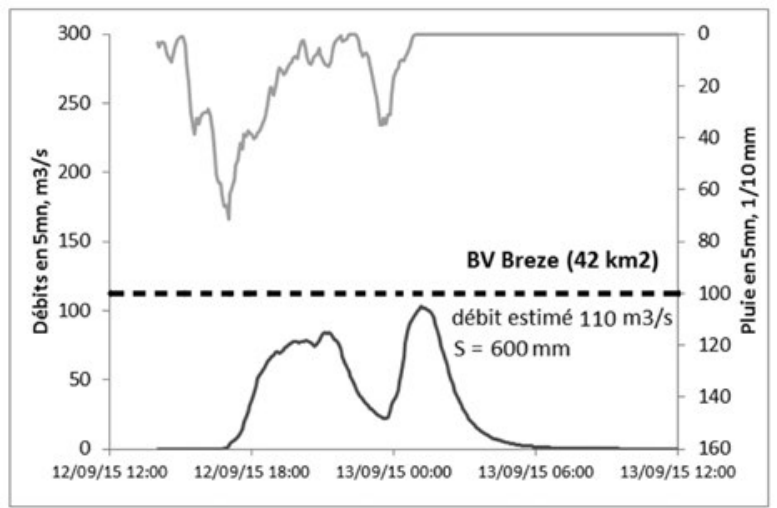

Figure 10 : Simulation de la crue à l'exutoire du bassin de la Brèze, à l'aide du modèle SCS-LR. Une valeur de $S=600 \mathrm{~mm}$ permet d'approcher le débit estimé à $110 \mathrm{~m}^{3} / \mathrm{s}$ par retour d'expérience. 


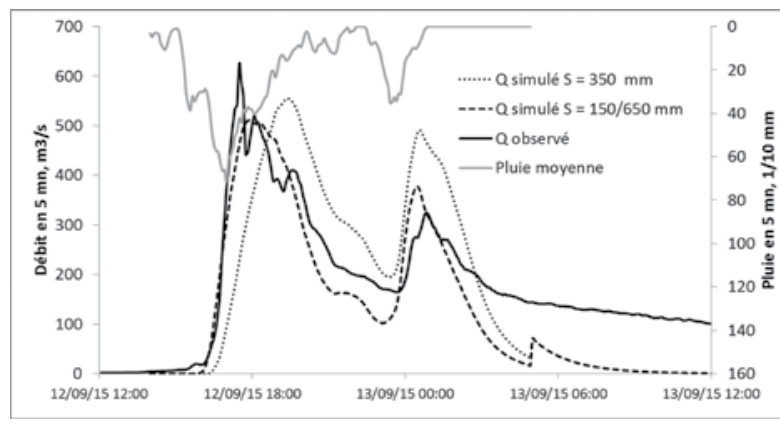

Figure 11 : Simulation de la crue de la Lergue à Lodève, à l'aide du modèle SCS-LR. Une valeur de $S=350 \mathrm{~mm}$ permet d'approcher le débit de pointe de crue estimé à $620 \mathrm{~m}^{3} / \mathrm{s}$ à partir de la hauteur d'eau mesurée et de la courbe d'étalonnage de la station. Une modèle à 2 classes de production $S=150 \mathrm{~mm}$ en aval et $S=650 \mathrm{~mm}$ en amont permet de mieux caler l'hydrogramme simulé dans le temps.

Laurounet). Des valeurs intermédiaires ont été obtenues pour les bassins mixtes : $300 \mathrm{~mm}$ pour la Lergue à Lodève (Fig. 11), station pour laquelle on dispose de l'hydrogramme complet de la crue, $400 \mathrm{~mm}$ pour Soulondre. Sur la figure 11, le retard des débits simulés est dû au fait que la production a été appliquée de façon uniforme sur l'ensemble du bassin, alors que la partie la plus productive du bassin est la partie aval. La simulation est ainsi améliorée en considérant un modèle à 2 classes de production, distinguant les zones karstiques ou non par l'altitude $370 \mathrm{~m}$, qui correspond approximativement à la limite d'extension des zones karstiques sur ce bassin (Fig. 6). Le paramètre de production $\mathrm{S}$ a été fixé à $150 \mathrm{~mm}$ pour la zone non karstique en aval (superficie $40.1 \mathrm{~km}^{2}$ ), et à $650 \mathrm{~mm}$ pour la zone karstique en amont (superficie $142.9 \mathrm{~km}^{2}$ ), en cohérence avec les valeurs de $\mathrm{S}$ obtenues pour les bassins à dominante karstique ou non-karstique.

Par ailleurs, et à titre de comparaison, les valeurs de $\mathrm{S}$ calées en utilisant la même approche sur un bassin comme le Gardon à Anduze (Gard), constitué essentiellement de schistes et de granites surmontés par des sols perméables, sont de l'ordre de 300-350 mm pour un épisode survenant sur des sols secs en début d'automne, et de 100-150 mm pour un épisode survenant sur des sols à demi-saturés [Tramblay et al., 2011]. Pour des conditions de saturation initiale des sols équivalentes à cet épisode, une valeur de S comprise entre 250 et $300 \mathrm{~mm}$ est probable, et situe la production du Gardon entre celle des pélites permiennes (100-200 mm) et celle des bassins à dominante karstique (600-700 mm).

\section{Comparaison avec autres retours d'expérience}

Afin de situer cet événement parmi les épisodes catastrophiques, nous avons repris les débits observés lors des REX conduits en France depuis une quinzaine d'années autour de la Méditerranée. La comparaison (Fig. 12) montre que l'événement du Gard du 8/9 septembre 2002 [Gaume et Bouvier, 2004, Delrieu et al, 2005, Brunet et al, 2012] domine largement les autres avec de nombreux débits spécifiques supérieurs aux débits rares de la formule Bressand Golossof, et proches de la courbe enveloppe établie pour l'Europe dans le cadre du projet européen Hydrate $\left(\mathrm{Q}_{\mathrm{m}^{3 / s}}=100 * \mathrm{~S} \mathrm{~km}_{2}{ }^{0.6}\right)$ [Gaume et al, 2009]. Les débits estimés de la crue de Lodève du 12/09/15 (pour laquelle on n'a retenu que les bassins de

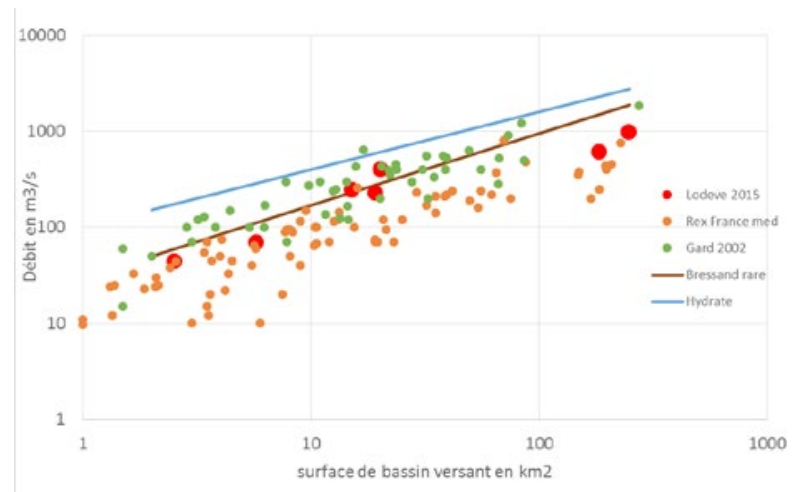

Figure 12 : Comparaison des débits estimés lors de différents REX en zone méditerranéenne française. Les points Rex France med proviennent des rex : Var 2010, Languedoc 2014 et Cote d'Azur 2015. Pour l'événement de Lodève, seuls les bassins versants de plaine ont été retenus

plaine) se situe dans la fourchette haute des autres événements Languedoc 2014, Var 2010 ou Côte d'Azur 2015.

\section{CONCLUSION}

L'événement hydrologique du 12 septembre 2015 sur le secteur de Lodève est un exemple intéressant de crue éclair méditerranéenne. Cette crue est la plus importante observée sur la Lergue à Lodève depuis le début des observations, en 1959. La nature contrastée des substrats géologiques des sous bassins versants a permis de mettre en évidence la capacité d'atténuation très nette des crues par le karst, dans ce cas précis. Les débits de pointe de crue estimés lors du retour d'expérience atteignent en effet $10-20 \mathrm{~m}^{3} / \mathrm{s} / \mathrm{km}^{2}$ pour les bassins situés à l'aval de Lodève, sur des terrains relativement imperméables, alors qu'ils ne sont que de $1-5 \mathrm{~m}^{3} / \mathrm{s} / \mathrm{km}^{2}$ pour les bassins situés sur les plateaux karstiques (dans des conditions de pluviométrie et de superficie de bassin relativement semblables). En considérant le cas de la Brèze, le débit spécifique de pointe de crue est divisé par 3, à superficie de bassin égale, ce qui traduit une réponse hydrologique atténuée de ces bassins karstiques, même en tenant compte des incertitudes d'estimation des débits. Le fort contraste des réponses hydrologiques des différents bassins versants touchés par l'épisode de 2015 est reflété par les valeurs calibrées du paramètre $\mathrm{S}$ du modèle de production SCS, 100 à $200 \mathrm{~mm}$ pour les bassins imperméables à l'aval de Lodève contre 600 à $700 \mathrm{~mm}$ pour les bassins karstiques à l'amont.

Par rapport à d'autres retours d'expérience, les débits observés sur les bassins versants de plaine se situent dans la fourchette haute des débits observés depuis 2002 (l'événement du Gard en 2002 restant au-dessus de tous les autres). Ceci confirme le caractère exceptionnel de cette crue non seulement pour le bassin versant de la Lergue, mais aussi à une échelle régionale.

\section{REMERCIEMENTS}

Les auteurs tiennent à remercier Arthur Marchandise (DREAL Occitanie) pour la fourniture des images radar Météo France Panthère ainsi que $\mathrm{S}$ Chédeville et $\mathrm{R}$ Muller pour leur aide indispensable dans la collecte des données de terrain. Ils remercient également O Payrastre, L Neppel, P-A Ayral et S Pistre pour la lecture du document et leurs conseils. 


\section{RÉFÉRENCES}

Brunet P, Bouvier C, Gaume E (2012) - Retour d'expériences sur les crues du Gard en septembre 2002 : contrôle de la qualité des débits estimés et comparaison avec les formules synthétiques régionales. Congrès SHF "Evénements extrêmes fluviaux et maritimes”, Paris, 1-2 février 2012.

Coustau M, Bouvier C, Borrell-Estupina, V, Jourde H (2012) Flood modelling with a distributed event-based parsimonious rainfall-runoff model: case of the karstic Lez river catchment. Nat. Hazards Earth Syst. Sci, 12, 1119-1133.

Creutin J.-D., Borga M., Lutoff C., Scolobig A., Ruin I. \& Creton-Cazanave L. (2009) - Catchment dynamics and social response during flash floods: the potential of radar rainfall monitoring for warning procedures. Meteorol. Appl, 16(1), 115-125.

Delrieu G, Ducroce V, Gaume E, Nicol J, Payrastre O, Yates E, Kirstetter P-E, Andrieu H, Ayral Pa, Bouvier C, Creutin Jd, Livet M, Anquetin A, Lang M, Neppel L, Obled C, Parent-Du-Chatelet J, Saulnier Gm, Walpersdorf A, Wobrock W. (2005) - The catastrophic flash-flood event of 8-9 September 2002 in the Gard region, France: a first case study for the Cevennes-Vivarais Mediterranean Hydro-meteorological Observatory. Journal of Hydrometeorology, 6, 34-52.

GAume E., Bouvier C. (2004) - Analyse hydro-pluviométrique des crues du Gard et du Vidourle des 8 et 9 septembre 2002. La Houille Blanche, 6, 99-106.

Gaume E., Borga M. (2008) - Post-flood field investigations in upland catchments after major flash floods: proposal of a methodology and illustrations. Journal of Flood Risk Management, 1, 175-189.

Gaume E., Bain V., Bernardara P., Newinger O., Barbuc M., Bateman A., BlašKovičová L., Blöschl G., Borga M., Dumitrescu A., Daliakopoulos I., Garcia J., Irimescu
A., Kohnova S., Koutroulis A., Marchi L., Matreata S., Medina V.,Preciso E., Sempere-Torres D., Stancalie G., Szolgay J., Tsanis I., Velasco D. \& Viglione A. (2009) A compilation of data on European flash floods. J. Hydrol, 367(1-2), 70-78.

Jourde H, Roesch A, Guinot V, Bailly-Comte V (2007) Dynamics and contribution of karst ground water to surface flow during Mediterranean Flood. Environmental Geology, 51(5), 725-730.

Marchandise A. (2007) - Modélisation hydrologique distribuée sur le Gardon d'Anduze ; étude comparative de différents modèles pluie-débit, extrapolation de la normale à l'extrême et tests d'hypothèses sur les processus hydrologiques. Thèse de Doctorat de l'Université de Montpellier, 194 pages + annexes.

Marechal J-C., Ladouche B., Dorflinger N. (2008) - Karst flash flooding in a Mediterranean karst, the example of Fontaine de Nîmes. Engineering Geology, 99, 138-146.

Meteo France. (1999) - Estimation des hauteurs de précipitations d'occurrence rare pour des durées de cumul de 1 à 10 jours sur 3000 postes français. Meteo France Juil 1999.

Payrastre O., E. Gaume P. Javelle B. Janet P. Fourmigue, Ph. Lefort A. Martin B. Boudevillain P. Brunet G. Delrieu L. Marchi Y. Aubert E. Dautrey L. Durand M. Lang L. Boissier J. Douvinet C. Martin I. Ruin Et L'equipe Tto2D D'HYMEX. (2012) - Hydrological analysis of the catastrophic flash flood of 15th June 2010 in the area of Draguignan (Var, France). Congrés SHF “Evénements extremes fluviaux et maritimes", Paris, 1-2 février 2012.

Tramblay Y., C. Bouvier P.-A. Ayral A. Marchandise (2011) Impact of rainfall spatial distribution on rainfall-runoff modelling efficiency and initial soil moisture conditions estimation. Nat. Hazards Earth Syst. Sci, 11, 157-170. 